\title{
Resección de la primera fila del carpo (Carpectomía Proximal)
}

\author{
I. Proubasta Renart, C. Lamas Gómez, J. Itarte Pujals, \\ J. De Caso Rodríguez.
}

Servicio de Cirugía Ortopédica y TRaumatología

Hospital de SANT PAU

AVDa. SAN ANTONio M $M^{a}$ CLARET, 167

O8025 BARCELONA

Correspondencia:

Dr. I. Proubasta

Servicio de Cirugía Ortopédica y Traumatología

Hospital de Sant Pau

Avda. San Antonio Ma Claret, 167

08025 Barcelona

Email: iproubasta@hsp.santpau.es

Se realizó un estudio retrospectivo de una serie de 12 pacientes a los que se les practicó la resección de la primera fila del carpo por diferentes patologías de la muñeca. Con un seguimiento medio de 1 año y 6 meses, se pudo observar que en el $92 \%$ de los casos se consiguieron buenos resultados, tanto clínicos como radiográficos, pues en tan sólo un caso se objetivaron cambios degenerativos entre la cabeza del hueso grande y la fosa semilunar de la carilla distal del radio. Los autores concluyen que la resección de la primera fila del carpo, siempre que la indicación sea correcta, constituye una buena alternativa a la artrodesis.

Palabras clave: Carpo, muñeca, SNAC, SLAC, Kienböck, carpectomía proximal.
A retrospective study of a series of 12 patients, who underwent proximal row carpectomy as a treatment for different wrist conditions, was carried out. With an average follow-up of 1 year and 6 months, it was observed that $92 \%$ of cases had good results, both radiographic and clinical, and only one case developed degenerative changes between the head of capitate and the lunate facet of the radius. The authors conclude that proximal row carpectomy, as long as it is indicated, is a good alternative to arthrodesis.

Key words: Carpus, wrist, SNAC, SLAC, Kienböck, proximal row carpectomy.

Rev. Iberam. Cir. Mano - Vol. 33 - Núm. 67 - Noviembre 2005 (22-30)

\section{INTRODUCCIÓN:}

a resección de la primera fila del carpo,
también llamada Carpectomía Proximal
$(\mathrm{CP})$, fue descrita por primera vez por

fermedad de Kienböck ${ }^{6-8}$. En estos 20 últimos años, las publicaciones al respecto muestran revisiones a largo plazo con resultados muy favorables $^{9-12}$. Sin embargo, existe un especial escepticismo en cuanto a la durabilidad de la CP en el tiempo, especialmente en lo concerniente al desarrollo de una artrosis radio-hueso grande. Sea como fuere, la CP constituye una intervención de rescate para salvaguardar y preservar al máximo la movilidad de la muñeca antes de proceder a su artrodesis ${ }^{13}$. 
Tabla I - Resultados obtenidos en NUESTRA SERIE DE 12 PACIENTES A LOS QUE SE les PRACticó UnA CARPeCtomía PROXIMAL (CP)

\begin{tabular}{|clllcll|}
\hline$N^{\circ}$ & Edad & Etiología & \multicolumn{2}{c}{ Movilidad } & Fuerza & Resultado \\
\hline 1 & & & FE & DC+DR & & \\
\hline 2 & 28 & SLAC & $80^{\circ}$ & $35^{\circ}$ & $80 \%$ & Excelente \\
\hline 3 & 24 & SLAC & $45^{\circ}$ & $30^{\circ}$ & $67 \%$ & Bueno \\
\hline 4 & 48 & SNAC & $60^{\circ}$ & $32^{\circ}$ & $77 \%$ & Excelente \\
\hline 5 & 33 & SNAC & $67^{\circ}$ & $40^{\circ}$ & $75 \%$ & Bueno \\
\hline 6 & 62 & SNAC & $50^{\circ}$ & $27^{\circ}$ & $60 \%$ & Bueno \\
\hline 7 & 40 & SNAC & $70^{\circ}$ & $43^{\circ}$ & $83 \%$ & Excelente \\
\hline 8 & 25 & LPC & $46^{\circ}$ & $23^{\circ}$ & $54 \%$ & Regular \\
\hline 9 & 33 & Kienböck & $44^{\circ}$ & $30^{\circ}$ & $85 \%$ & Excelente \\
\hline 10 & 46 & Kienböck & $40^{\circ}$ & $33^{\circ}$ & $81 \%$ & Bueno \\
\hline 11 & 26 & Kienböck & $42^{\circ}$ & $31^{\circ}$ & $65 \%$ & Bueno \\
\hline 12 & 28 & Kienböck & $66 \%$ & $40^{\circ} \%$ & $56 \%$ & Bueno \\
\hline
\end{tabular}

LPC: Luxación Perilunar Carpo; FE: flexoextensión. DC + DR: desviación cubital + desviación radial.

El propósito de este trabajo, es revisar retrospectivamente los resultados obtenidos en una serie consecutiva de 12 pacientes a los que se les practicó una $\mathrm{CP}$.

\section{MATERIAL Y MÉTODO}

Desde enero de 1999 hasta enero de 2003, la resección de la primera fila del carpo fue practicada en 12 pacientes, todos varones, con una edad media de 43 años (rango: 19-54 años). En 10 casos la mano intervenida fue la derecha, que coincidió también con la mano dominante, mientras que en los 2 casos restantes fue la izquierda y no dominante. En cuanto a la profesión de los pacientes, siete eran comerciantes, dos administrativos y los otros tres restantes, sin una actividad definida. El seguimiento de estos pacientes fue de 1 año y 6 meses de media, con un mínimo de 1 año y 4 meses a un máximo de 4 años. En cuanto a las causas por las que se procedió a la $\mathrm{CP}$, se observó que en 4 casos existía una enfermedad de Kienböck (todos en estadío III de Litchman), en 4 una muñeca SNAC (3 en estadío I y 1 en estadío II), en 3 una muñeca SLAC (todos en estadío II), y en 1 caso se objetivó una luxación perilunar del carpo inveterada.

El tiempo medio entre el inicio de los síntomas y/o del traumatismo productor de la lesión fue de 7 meses, variando entre 3 meses y 3 años.

Todos los pacientes fueron controlados clínica y radiográficamente. Al respecto, se valoró la pérdida de relación articular entre la fosa semilunar de la carilla articular del radio con la cabeza del hueso grande así como la existencia de cambios degenerativos a dicho nivel, y por lo que hace referencia al estudio clínico, se siguieron los criterios de Minami y cols. ${ }^{13}$, los cuales tienen en cuenta el dolor, la movilidad y la fuerza de prensión (Tabla I). En cuanto a la movilidad, se midieron radiográficamente los 


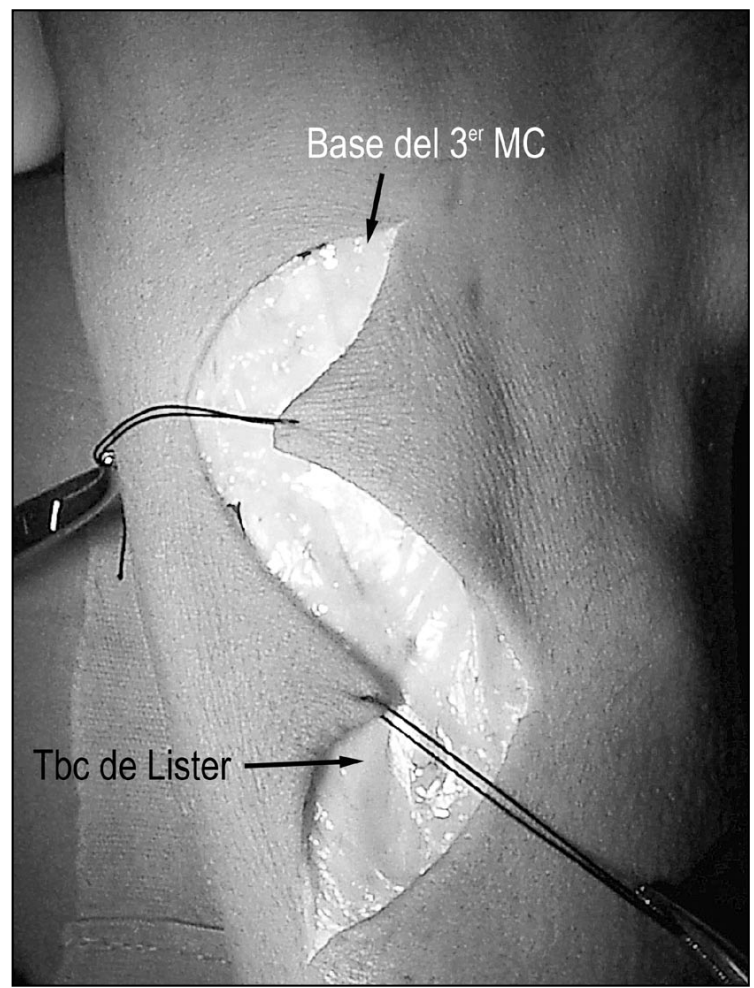

Figura 1. Incisión de piel.

ángulos máximos de extensión, flexión, desviación cubital y desviación radial, a través de los ejes longitudinales formados por el radio y el hueso grande, mientras que la fuerza de prensión fue medida con un dinamómetro JAMAR ${ }^{\circledR}$, transformando el valor hallado en porcentaje con respecto al valor normal obtenido en la muñeca contralateral.

\section{TÉCNICA QUIRÚRGICA}

En todos los casos se siguió el mismo procedimiento quirúrgico. Así, bajo manguito neumático a $250 \mathrm{mmHg}$ y previa exanguinación del brazo, se inicia la intervención mediante incisión dorsal en zig-zag sobre la muñeca, de tal manera que la zona proximal de la incisión esté a $1 \mathrm{~cm}$ proximal al tubérculo de Lister y que la zona distal de la misma acabe a nivel de la base del tercer metacarpiano (Figura 1). Dicha incisión la empleamos habitualmente por ser más versátil que la incisión longitudinal y/o transversal, y también porque facilita cualquier cirugía adicional si ésta fuera necesaria. Después de disecar cuidadosamente los dos colga- jos de piel para evitar lesionar las ramas sensitivas de los nervios radial y cubital, se secciona perpendicularmente el retináculo extensor a nivel del tercer compartimento. Para exponer la cápsula articular radiocarpiana dorsal, se luxa y retrae el tendón del extensor largo del pulgar (extensor pollicis longus) y los extensores del carpo (extensor carpi radialis longus y brevis) hacia el lado radial, mientras que el extensor propio del dedo indice (extensor indicis propius) y el extensor común de los dedos (extensor digitorum comunis) se retraen cubitalmente. Debajo mismo del extensor propio del dedo índice y discurriendo paralelo al borde cubital del radio se localiza y se secciona el nervio interóseo posterior como medida antiálgica adicional. Después de proceder a identificar la articulación radiocarpiana mediante movimientos de flexoextensión, se practica una capsulotomía en «T» invertida, con un segmento axial centrado sobre el hueso grande y un trazo transversal desde la estiloides radial hasta la articulación radiocubital distal, procurando dejar un segmento capsular de unos 2-3 mm para su posterior cierre. Una vez practicada la capsulotomía, es fácil observar las dos interlíneas carpianas, la radiocarpiana y la mediocarpiana, respectivamente. Mediante la flexión y distracción de la muñeca, se comprueba el estado de las superficies cartilaginosas de la cabeza del hueso grande y de la fosa semilunar del radio. En el caso de existir cambios degenerativos significativos de dichas superficies articulares, se contraindica la $\mathrm{CP}$ y se procede a efectuar otra técnica quirúrgica alternativa como la artrodesis parcial radiocarpiana ó intercarpiana y/o la artrodesis total de muñeca. En caso de estar indemnes las superficies cartilaginosas comentadas, se inicia la CP formalmente. Esta comprende la exéresis total del piramidal, semilunar y escafoides. Durante este tiempo quirúrgico, es aconsejable realizar continuos movimientos de distracción manual de la muñeca con el fin de facilitar las resecciones óseas. No somos partidarios de efectuar en bloque la extirpación de los huesos de la primera fila por cuanto es muy dificultoso y potencialmente peligroso, especialmente a nivel del polo distal del escafoides, a cuyo nivel discurre la arteria radial. Si bien no importa el orden en que se procede a la exére- 


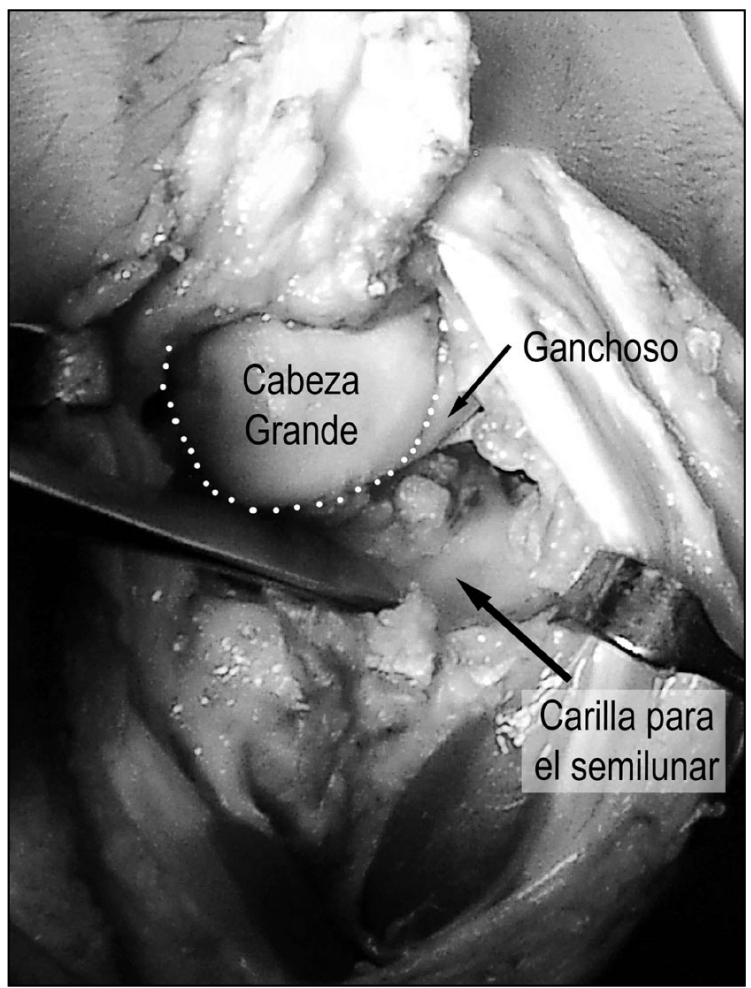

Figura 2. Una vez resecada la primera fila del carpo, debe observarse la cabeza del hueso grande, el hueso ganchoso, la superficie articular del radio con sus fosas independientes del semilunar y escafoides.

sis de los huesos de la primera fila, nosotros la iniciamos por el polo proximal del escafoides y siempre por medio de pinza gubia. A continuación seguimos con el semilunar y el piramidal, y la finalizamos con la exéresis del polo distal del escafoides, aunque dejamos siempre su porción articular con el trapecio para evitar la lesión del ligamento radioescafoideo. Este fragmento de hueso, aunque radiográficamente es visible, no afecta negativamente el resultado clínico. Sin embargo, si dicho fragmento es demasiado grande, puede chocar con la estiloides radial y, en consecuencia, provocar un roce contínuo. En estos casos está justificada la estiloidectomía ${ }^{14}$. Un truco que puede ser útil para valorar si existe o no un roce anómalo, es colocar el pulpejo del dedo meñique en el espacio entre la apófisis estiloides del radio y el fragmento escafoideo, y determinar si éste es suficientemente amplio como para permitir el movimiento comentado sin producir contacto.

Una vez realizada la $\mathrm{CP}$, en el campo quirúrgico debe verse: la superficie articular del ra-

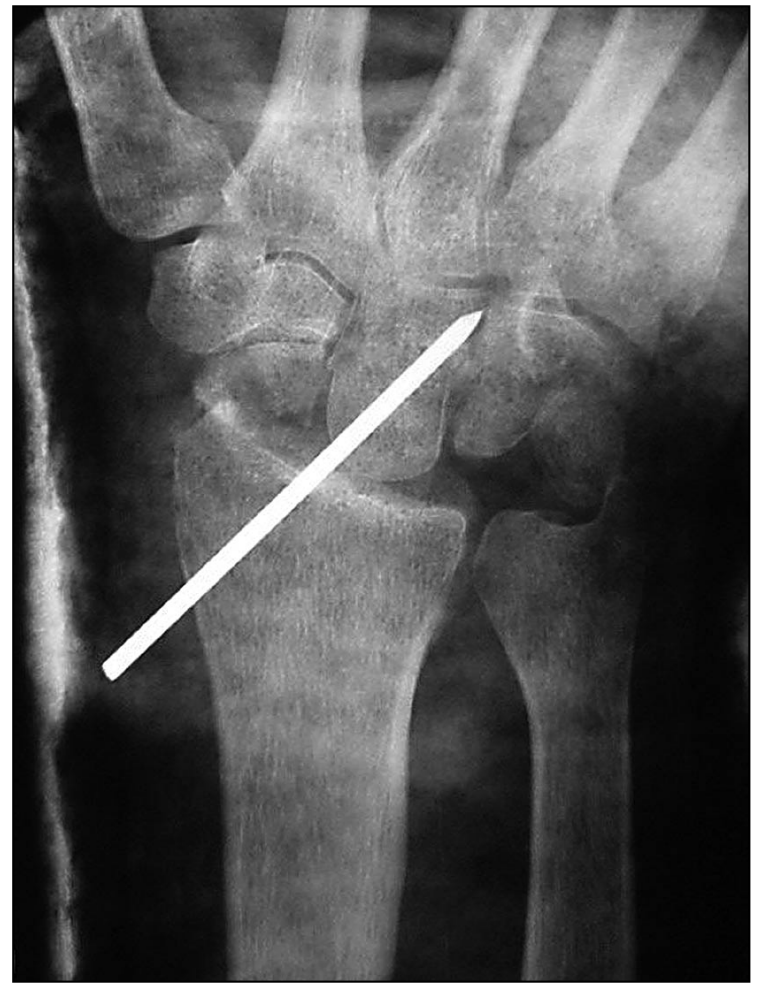

Figura 3. Estabilización temporal del hueso grande con el radio mediante aguja de Kischner. Obsérvese que la aguja no atraviesa las superficies articulares del radio con el hueso grande para evitar un daño innecesario.

dio con sus dos fosas independientes, la del semilunar y la del escafoides, la cabeza del hueso grande, el hueso ganchoso, la superficie cartilaginosa del pisiforme y la porción ósea distal del escafoides (Figura 2). Seguidamente, se adaptan las superficies articulares de la cabeza del hueso grande y la correspondiente a la fosa semilunar del radio, comprobando su buena adaptación. Finalmente, el campo quirúrgico es debidamente irrigado con suero fisiológico y se procede a suturar la cápsula articular. En este sentido, como se ha resecado la primera fila del carpo, existe un acortamiento de la muñeca y, en consecuencia, un exceso de cápsula articular. Por tanto, es preciso resecar parte de la misma a nivel proximal de los colgajos capsulares distales para poder efectuar su anclaje al radio con la tensión necesaria pero permitiendo siempre conservar un espacio laxo entre la superficie del radio y la segunda fila del carpo. Dicha sutura se efectúa con hilo reabsorbible de 3/0 (Vicryl $\left.{ }^{\circledR}\right)$ con la muñeca en ligera extensión $\left(10^{\circ}-15^{\circ}\right)$ y desviación radial $\left(15^{\circ}\right)$. Esta posi- 
Resección de la primera Flla del Carpo (Carpectomía ProXimal)
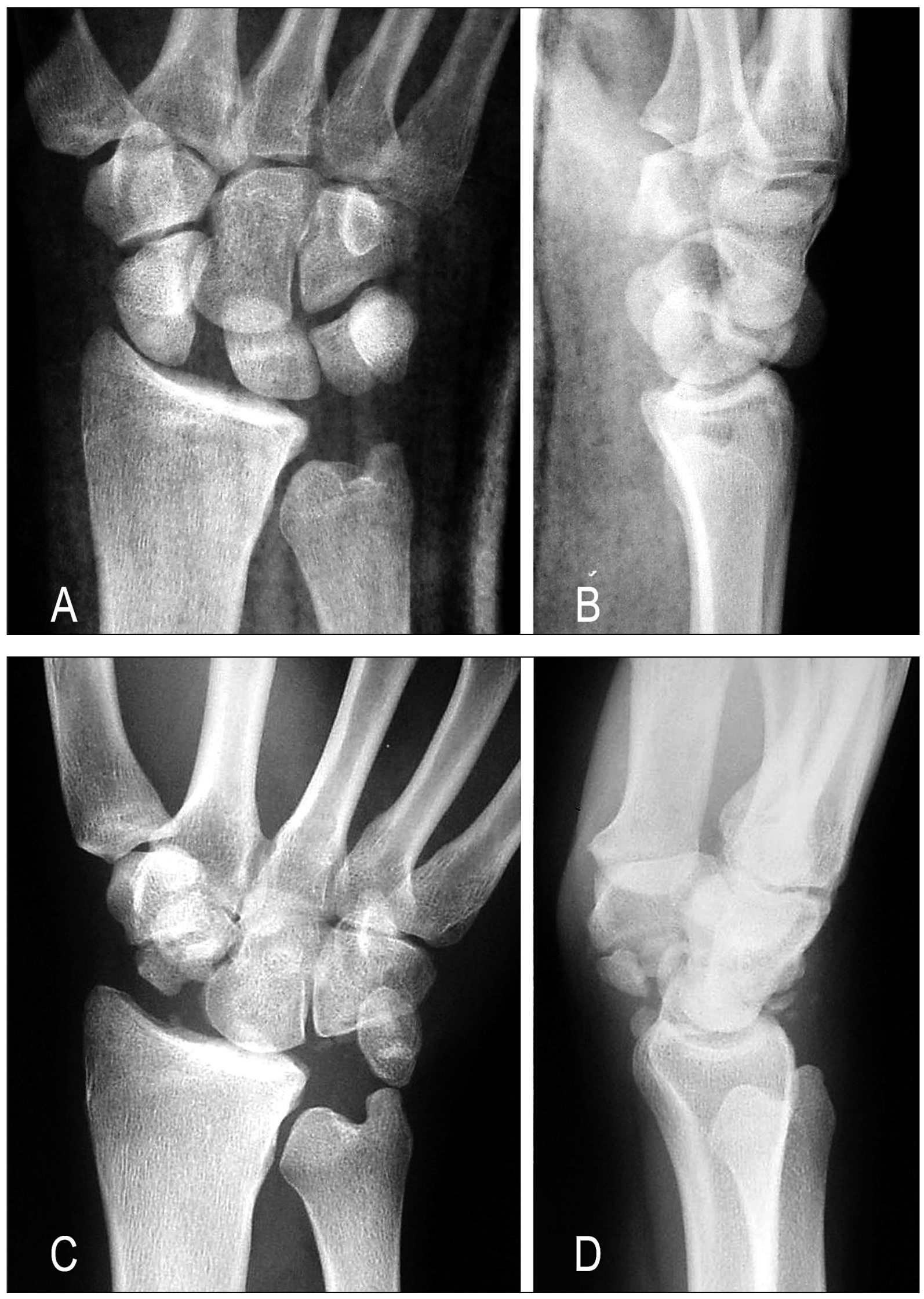

Figura 4. Estudio radiográfico pre y postoperatorio tras una CP en un paciente con muñeca SLAC tipo I. 
Tabla II - Criterios clínicos según Minami y cols (15)

\begin{tabular}{|lccc|}
\hline Clasificación & Dolor & Movilidad & Fuerza \\
\hline Excelente & NO & $50 \%$ & $>70 \%$ \\
\hline Bueno & Ligero 30\% & $50-70 \%$ & \\
\hline Regular & Moderado & $20 \%$ & $30-50 \%$ \\
\hline Malo & Severo & $<20 \%$ & $<30 \%$ \\
\hline
\end{tabular}

ción permite un óptimo posicionamiento de la cabeza del hueso grande dentro de la fosa semilunar del radio. A continuación se sutura el retináculo extensor y la piel con Prolene 5/0. Finalmente, se coloca un yeso tipo escafoides con la muñeca en aproximadamente $15^{\circ}$ de extensión y desviación radial.

En ocasiones, nos podemos encontrar que la cabeza del hueso grande no se recoloca adecuadamente en la fosa semilunar del radio, en cuyo caso es prudente sintetizar temporalmente el radio al hueso grande con una aguja de Kirschner de 1,5 mm de diámetro, con el fin de evitar su desplazamiento hacia la fosa escafoidea de la superficie articular del radio. Dicho enclavado lo realizamos por fuera de la superficie articular de la cabeza del hueso grande para no lesionar su superficie cartilaginosa (Figura 3). Finalizado el periodo de inmovilización, se retira la aguja para iniciar el movimiento de la muñeca. Asimismo, durante la exéresis de los huesos carpianos, el cirujano debe tener mucho cuidado en no dañar las superficies articulares de la fosa del semilunar del radio y la del hueso grande, pues gran parte del éxito del resultado obtenido depende de la integridad cartilaginosa de los elementos óseos comentados.

En el postoperatorio, durante las primeras 24 horas, el paciente debe llevar su mano en cabestrillo a la altura del corazón y movilizar activamente los dedos de la mano. A partir de entonces, se permite también la movilidad activa y sin restricciones del codo y hombro. El estudio radiográfico con proyecciones posteroanterior y lateral, permite objetivar el correcto posicionamiento de la cabeza del hueso grande dentro de la fosa semilunar del radio, en ambos planos (Figura 4). En el caso de existir una pérdida de la reducción, deberá reintervenirse inmediatamente al paciente para conseguir una perfecta congruencia. En estos casos está plenamente justificado la síntesis temporal del radio con el hueso grande mediante una aguja de Kirschner, si no se realizó ya previamente. De no existir complicaciones, a las dos semanas se retiran los puntos y se pasa a un yeso tipo Colles durante dos semanas más. En total pues, 4 semanas de inmovilización, a partir de las cuales se permite la movilización libre y activa de la muñeca (Figura 5). Sin embargo, es aconsejable que durante las siguientes 2-3 semanas, el paciente utilice una férula de descanso por la noche, pues con ello se evitan posturas forzadas y se facilita la disminución de la inflamación.

\section{RESULTADOS}

Excepto en un caso, en que se objetivaron cambios degenerativos entre la cabeza del hueso grande y el radio y que correspondió al paciente con una luxación perilunar inveterada del carpo, en los demás pacientes no se constató ningún signo degenerativo a dicho nivel. La movilidad media observada de flexoextensión fue de 56,7ํ (máxima: 90ㅜㅜ mínima: 35ํ), mientras que la desviación cubital+desviación radial fue de 32,9o (máxima: 36o mínima: 23ํ) y, en cuanto a la fuerza, se obtuvo un valor medio del 73\% (máxima: 85\%; mínima: 54\%) con respecto a la contralateral (Tabla II). 

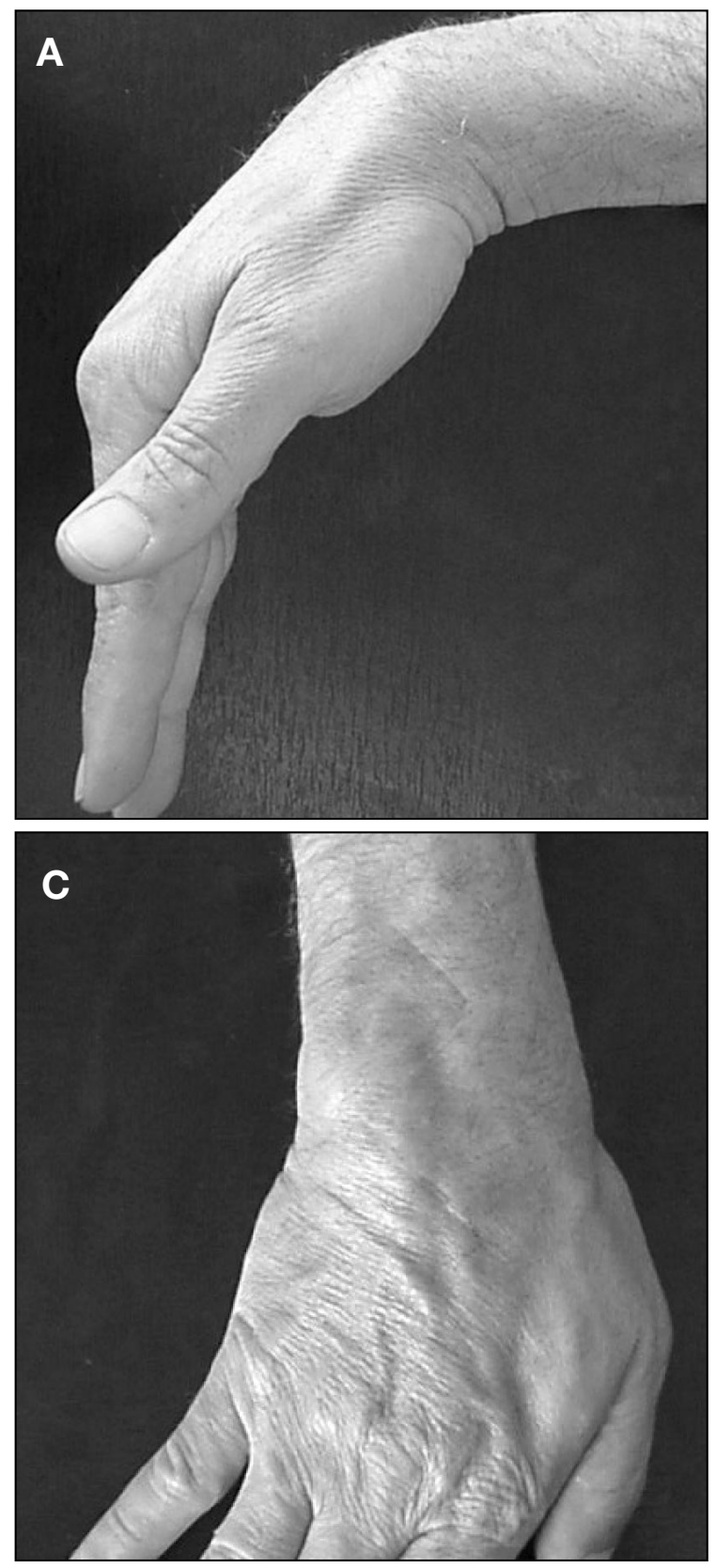

Figura 5. Movilidad de la muñeca, a los 6 meses de practicada una CP. A) Flexión. B) Extensión. C) Desviación cubital. D) Desviación radial.

\section{DISCUSIÓN}

Desde que en 1944, Stamm ${ }^{1}$ describiera la técnica quirúrgica denominada Carpectomía Proximal $(\mathrm{CP})$, muy poco se ha estudiado del porqué dicha intervención ha tenido resultados muy favorables, especialmente en estos últimos años donde los estudios a largo plazo han mostrado que la movilidad articular de la muñeca
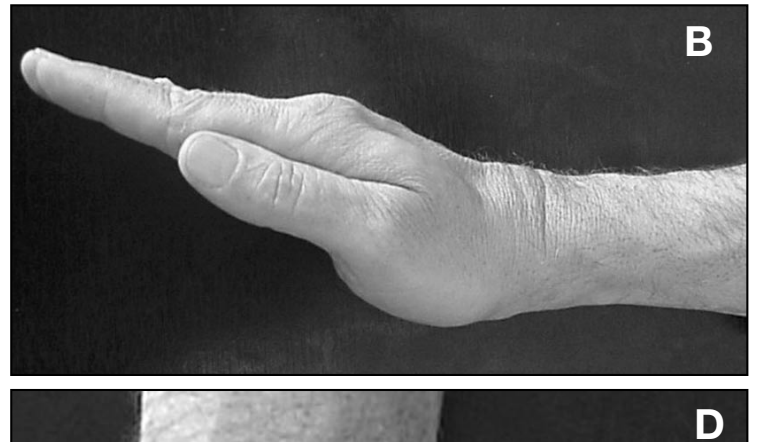

D es más que aceptable, que la fuerza de prensión, aunque disminuida, se mantiene en el tiempo y, lo que es más importante, el dolor desaparece y/o disminuye considerablemente hasta el punto de que en un gran porcentaje de casos se vuelve de nuevo al trabajo original que el paciente ejercía antes de la intervención. Los resultados observados en la literatura así como los obtenidos en nuestra serie, avalan lo 
comentado, al menos en lo que se refiere a la fuerza de prensión y al movimiento combinado de desviación cubital + desviación radial. Sin embargo, por lo que respecta al movimiento combinado de flexoextensión, la revisión de la bibliografía muestra que el valor medio obtenido es de unos $72^{\mathrm{o} 15}$, cifra superior a la observada en nuestra serie, que sólo fue de 56,7 ${ }^{\circ}$ de media. La etiología de tal hecho es controvertida, si bien apuntamos dos posibles causas: la primera sería le tensión excesiva de la cápsula articular y, la segunda, el tiempo de inmovilización. En este último aspecto, quizás la inmovilización de dos semanas sería suficiente para prevenir dicho problema. En cualquier caso, el rango de movimiento obtenido en ambos planos, permiten realizar al paciente el $90 \%$ de las actividades diarias sin merma importante de la función ${ }^{16}$.

Por todo ello, aún considerando que la carpectomía proximal es una cirugía relativamente mutilante, constituye una alternativa muy válida antes de proceder con otro tipo de intervenciones, como son las artrodesis parciales del carpo (cuatro esquinas) e incluso las denervaciones de muñeca ${ }^{17}$. Con respecto a las artrodesis parciales del carpo y, en concreto la denominada «cuatro esquinas», estudios comparativos entre este tipo de artrodesis y la carpectomía proximal, no muestran diferencias significativas en cuanto al resultado se refiere, aunque indican que con la carpectomía se presentan menos complicaciones y es técnicamente más fácil ${ }^{18,19}$. Sin embargo, al igual que en toda técnica quirúrgica, es preciso que la indicación de una CP sea correcta. En este sentido y según nuestra corta experiencia, es requisito fundamental, que tanto el cartílago articular de la cabeza del hueso grande como el de la fosa semilunar del radio no presenten ningún proceso degenerativo, pues de lo contrario, el resultado es irremediablemente malo. No obstante, hay autores que consideran que las lesiones condrales de la cabeza del hueso grande de menos de $0,5 \mathrm{~mm}$, no son una contraindicación absoluta, pues reportan algunos casos con excelentes resultados ${ }^{20}$. En nuestra serie, el único caso con resultado desfavorable y que correspondió también al paciente con una luxación perilunar inveterada del carpo, se ob- servó que existía ya un defecto cartilaginoso de unos $4 \mathrm{~mm}$ de diámetro en la cabeza del hueso grande y otro más difuso en el márgen dosal de la carilla articular del radio. Y por lo que respecta a las denervaciones de la muñeca, los resultados son bastantes aleatorios ${ }^{17}$; quizás, su única indicación sería en aquellos pacientes con poca demanda funcional. Así pues, cualquier patología de la muñeca que cumpla la premisa de tener conservadas las superficies articulares de la cabeza del hueso grande y la fosa semilunar del radio, puede ser candidata a una CP. En este contexto, procesos tales como la artrosis tipo SLAC o SNAC en estadíos I y II, la enfermedad de Kienböck en estadíos II y III de Litchman, las fracturas-luxaciones y/o luxaciones perilunares del carpo, agudas o crónicas, son algunas de las indicaciones para realizar dicha técnica. $\mathrm{Al}$ respecto, si bien la mayoría de casos pueden abordarse adecuadamente por vía dorsal, hay algún autor que preconiza la vía palmar, especialmente en aquellos pacientes con luxación inveterada del semilunar $^{21}$. Asimismo comentar que hay autores que efectúan la carpectomía por vía artroscópica $^{22}$, de la cual no tenemos experiencia alguna.

Finalmente indicar que, en el caso de deterioro articular entre la cabeza del hueso grande y el radio, no hay problema en realizar una artrodesis de muñeca, pues se ha constatado que proporcionan unos resultados similares a una artrodesis convencional ${ }^{23}$. En nuestra serie, en ningún caso se tuvo que practicar tal proceder al final del estudio.

\section{CONCLUSIONES}

- La Carpectomía proximal (CP), constituye una intervención de rescate que consigue preservar un rango de movimiento funcional.

- Las indicaciones fundamentales para proceder a una $\mathrm{CP}$ son múltiples, entre las que cabe citar el colapso escafolunar avanzado (SLAC) en estadíos I y II, el colapso avanzado por pseudoartrosis del escafoides carpiano (SNAC) en estadíos I y II, la enfermedad de Kienböck en estadíos II y III de Litchman, y en las fracturas-luxaciones y/o luxaciones peri- 
lunares del carpo inveteradas. De hecho, las tres primeras patologías suman el $72 \%$ de los casos publicados. En nuestra serie, dicho porcentaje fue del $91,7 \%$.

- En nuestra experiencia, el único requisito que se precisa para realizar una $\mathrm{CP}$, es que los cartílagos articulares de la cabeza del hueso grande y de la fosa semilunar del radio estén conservadas.

- El porcentaje de excelentes y buenos resultados, se sitúa entre el 70 al $100 \%$ de los casos, a corto plazo. En nuestra serie se obtuvo en 11 de los 12 pacientes, es decir, en el 91,7\% de los casos.
1. Stamm, T. T.: Escisión of the proximal row of the carpus. Proc R Soc Med 1944; 38: 74-5.

2. Arcalís, A. A.; Pedemonte, J. J.; Massons, J. A.: Artroplastia de resección de la primera hilera del carpo en las formas evolucionadas de pseudartrosis del escafoides carpiano. Rev Ortop Traum 1998; 42, Suppl 1: 39-41.

3. Inglis, A. E.; Jones, E. C.: Proximal-Row Carpectomy for Diseases of the Proximal Row. J. Bone Joint Surg 1977; 59A: 460-3.

4. Siegert, J. J.; Frassica, F. J.; Amadio, P. C.: Treatment of chronic perilunate dislocations. J. Hand Surg 1988; 13A: 206212.

5. Inoue, G.; Shionoya, K.: Late treatment of unreduced perilunate dislocations. J. Hand Surg 1999; 24B: 221-5.

6. Begley, B. W.; Engber, W. D.: Proximal row carpectomy in advanced Kienböck's disease. J. Hand Surg 1994; 19A: 1016-8.

7. Lin, H. H.; Stern, P. J.: «Salvage» procedures in the treatment of Kienböck's disease. Proximal row carpectomy and total wrist arthrodesis. Hand Clin 1993; 9: 521-6.

8. Nakamura, R.; Horri, E.; Watanabe, K.; Nakao, E.; Kato, H.; Tsunoda, K.: Proximal row carpectomy versus limited wrist arthrodesis for advanced Kien-

\section{BIBLIOGRAFÍA}

böck disease. J. Hand Surg 1998; 23B: 741-5.

9. Retting, M. E.; Raskin, K. B.: Long-term assessment of proximal row carpectomy for chronic perilunate dislocations. J. Hand Surg 1999; 24A: 1231-36.

10. Schernberg, F.; Nurbel, B.; Harisboure, A.; Lawane, M.: Resection of the first row of the carpus: results in 44 cases at moore that ten years. J. Bone Joint Surg; 2002; Suppl 1: 48.

11. Tomaino, M. M.; Delsignore, J.; Burton, R. I.: Long-term following proximal row carpectomy. J. Hand Surg 1994; 19A: 694703.

12. DiDonna, M. L.; Kiefhaber, T. R.; Stern, P. J.: Proximal row carpectomy: study with a minimum of ten years of followup. J. Bone Joint Surg 2004; 86-A: 2359-65.

13. Minami, A.; Agino, T.; Minami, M.: Limited wrist fusions. J Hand Surg 1988; 13A: 600-7.

14. Green, D. P.: Proximal row carpectomy. Hand Clin 1987; 3: 163-8.

15. Culp, R. W.; McGuigan, F. X.; Turner, M. A.; Lichtman, D. M.; Osterman, A. L.; McCarroll, H. R.: Proximal row carpectomy: a multicenter study. J. Hand Surg 1993; 18A: 19-25.

16. Nelson, D. L.: Functional wrist motion. Hand Clin 1997; 13: 83-92.
17. Tomaino, M. M.; Miller, R. J.; Cole, I.; Burton, R. I.: Scapholunate advanced collapse wrist: proximal row carpectomy or limited wrist arthrodesis with scaphoid excision? J. Hand Surg 1994; 19A: 134-42.

18. Saffar, P.; Fakhoury, B.: Résection de la première rangée contre arthrodèse partielle des os du carpe dans les instabilités du carpe. Ann Chir Main Memb Super 1992, 11, 276-280.

19. Wyrick, J. D.; Stern, P. J; Kiefhaber, T. R.: Motion-preserving procedures in the treatment of scapholunate advanced collapse wrist: proximal row carpectomy versus fourcorner arthrodesis. J. Hand Surg 1995; 20A: 965-70.

20. Imbriglia, J. E.; Broudy, A. S.; Hagberg, W. C.; McKernan, D.: Proximal row carpectomy: clinical evaluation. J. Hand Surg 1990; 15A: 426-30.

21. Luchetti, R.; Soragni, O.; Fairplay, T.: Proximal row carpectomy through a palmar approach. J. Hand Surg 1998; 23B: 406-9.

22. Culp, R. W.; Osterman, A. L.; Talsania, J. S.: Arthroscopic Proximal Row Carpectomy. Tech Hand Upper Extrem Surg 1997; 1: 116-19.

23. Richards, R. S.; Roth, J. H.: Simultaneous proximal row carpectomy and radius to distal carpal row arthrodesis. J Hand Surg 1994; 19A: 728-32. 